\title{
CALCULATING ANCESTRAL INFLUENCE IN MAN: A MATHEMATICAL MEASURE OF THE FACTS OF BISEXUAL HEREDITY 1
}

\author{
HARRY H. LAUGHLIN \\ Eugenics Record Office, Cold Spring Harbor, Long Island, New York \\ [Received April 24, 1920]
}

According to the common method of calculating ancestral influence in man, the germ-plasm is considered as an indefinitely dilutable substance contributed equally by the father and mother in making up the hereditary constitution of the offspring. Barring special phenomena, the several direct ancestors in each generation are, among them, quite properly accredited with the possession, patently or latently, of all of the traits of a particular offspring. In calculating the influence of a given ancestor in contributing toward the whole body of these traits in the offspring, the measure is a quotient determined by dividing unity by the number of direct ancestors in the given generation. Thus, the ancestral influence of one of the four grandparents is commonly said to be one-fourth, that of one of the eight great-grandparents one-eighth, and so on.

Sir Francis Galton, after a very careful study of the inheritance of human stature, proposed a modification of this orthodox notion of ancestral influence. His scheme is more in consonance with many of the observed facts than is the common method of dividing unity by the number of direct ancestors in the particular generation. His rule, or GaLton's Law as it is sometimes called, ascribes one-half of all ancestral influence to the two parents (each parent one-half of $5 \circ$ percent, or 25 percent), onefourth to the four grandparents (each grandparent one-fourth of 25 percent, or 6.25 percent), one-eighth to the eight great-grandparents (each great-grandparent one-eighth of $I 2.5$ percent, or $I .5625$ percent). Later Karl Pearson made some mathematical refinements of this scheme. But the study of pedigrees is so replete with cases showing the segregation and re-combination of ancestral traits that, even when traits are massed and averaged, neither the common quotients of the genealogists nor the generalizations of GaLton or Pearson suffice to explain the pedigree-facts, nor does either scheme square with the cytological evidence in the case.

\footnotetext{
${ }^{1}$ Read before the National Academy of Sciences, November io, rgig, at New Haven, Connecticut.
} 
With the rise of Mendelian investigations and the discovery of the close co-ordination between Mendelian pedigree-findings and the cytological evidence of the mechanism of heredity, there was founded the modern science of heredity, which is much more generally consistent with the observed facts than are any of the older systems of gauging natural inheritance.

By ancestral influence is meant the contribution of definite hereditary mental, physical and moral qualities, by a given ancestor to a given descendant. Pedigree-studies have set forth in man, as in many other organisms, rules of inheritance of many specific traits or qualities. Cytological investigations have shown how chromosomes duplicate, segregate and recombine (sometimes crossing-over and showing other special phenomena) in the course of gametogenesis, fertilization and development.

Neither the human nor any other germ-plasm is indefinitely dilutable or divisible, as many genealogists have assumed, but the dilutability and divisibility of the germ-plasm are limited by the number of chromosomes characteristic of the gametes of the particular species. In the human zygote two gametes, each with i2 chromosomes, unite. ${ }^{2}$ This means that, barring crossing-over, mutation, and other special phenomena, normally the human germ-plasm, and consequently all hereditary human qualities, that is, the "blood," is divisible only into 24 uneven units corresponding to the 24 zygotic chromosomes.

It is well demonstrated that the chromosomes are composed of genes or the determiners of natural inheritance. Each chromosome is a generadical or linkage-group of trait determiners. If then it is sought to measure the influence of a given ancestor upon the hereditary traits of a given propositus, it is necessary to develop a series of mathematical formulae which measure the probabilities of definite situations happening in reference to the transmission of chromosomes from the given ancestor to the given propositus.

In developing such formulae the following factors must be taken into consideration:

First. Whether the species in question is bisexual.

\footnotetext{
${ }^{2}$ There is still disagreement among cytologists concerning the number of chromosomes in man. VON WINIWARTER ( 1 III 2 ) concludes that the zygotic number of chromosomes in the human male is 47 , in the human female 48 , while Werman ( $\operatorname{IgI}_{7}$ ) concludes that the zygotic or diploid number of chromosomes is 24 , of which 2 are the $\mathrm{XY}$ idiochromosomes. When this problem is settled beyond peradventure, the correct number can be used in the accompanying formulae, which are here stated in general terms. For a history of the cytological study of the chromosome number in man see the excellent analytical bibliography given by ETHEe Browne Harvey in Journal of Morphology 34: 47-49, June, 1920.
} 
Second. Whether in the particular species the male or the female is the heterozygous sex.

Third. Due provision must be made for sex-linked traits.

Fourth. The number of chromosomes, both gametic and zygotic, characteristic of the species, must be known.

Fifth. For the present the individual chromosomes will not be weighted, but simply counted. Doubtless the ontogenetic working out of each chromosome is very specific both qualitatively and quantitatively. When ultimately genetical and cytological research locate in definite chromosomes the genes for definite traits, then the several chromosomes can be weighted, and due provision for their differential values properly made in the formulae for measuring the several features of ancestral influence.

Sixth. Due cognizance must be taken of the possibility of mutation, crossing-over, non-disjunction and other special chromosomal phenomena occurring in the course of the descent of chromosomes from a given ancestor to the propositus. In the accompanying formulae such phenomena are not provided for, because their rates of occurrence are not yet measurable in man, but when one by one their mathematical measures are attained, each such measure can be woven into the foundation formulae here given. The present formulae will not be upset by such corrections, but simply modified in the direction of greater accuracy.

Seventh. It must be considered that in a given gamete a definite chromosome may have been derived from either the paternal or the maternal source. This is especially important in cases of zygotes of mixed or mongrel blood.

Eighth. In certain situations it will be necessary to specify whether a given trait is dominant or recessive to its allelomorph.

Finally, all formulae must be stated in general terms, so that they may apply with equal accuracy to any species of plant or animal concerning which chromosomal data are at hand.

Applying these principles to man, the accompanying charts set forth in pedigree-fashion and measure mathematically a few selected definite features of the hereditary influence of each particular ancestor taken as a type, upon the propositus or $F_{1}$ zygote. These calculations are based upon the following data and conditions: (a) that the gametic number of human chomosomes is $\mathrm{I} 2$, the zygotic or somatic number in each sex 24 ; (b) that the male is heterozygous (XY) and the female homozygous (XX) in reference to sex; (c) that all of the purely hereditary qualities are carried by the several chromosomes; and (d) that in the first or foundation formulae no provision will be made for mutation, crossing-over, non-disjunction and other special phenomena. 


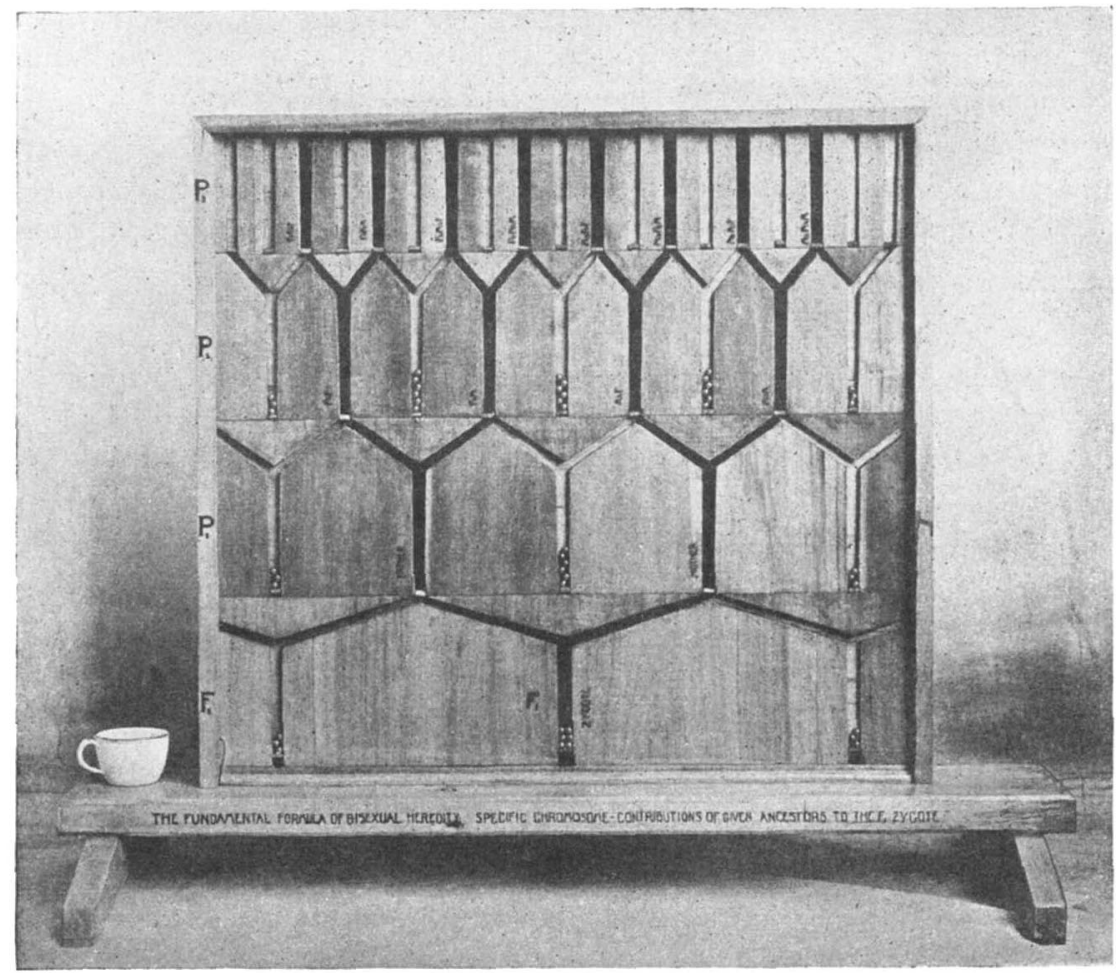

FigurE I.-Mechanism showing the essential features of segregation and recombination in the transmission of chromosomes from ancestors to descendants.

In the case here pictured, as shown by the location of the balls, the descent of chromosomes is traced in a bisexual species characterized by three pairs of chromosomes. Into each of the eight slots representing the eight ancestors of the $\mathrm{P}_{3}$ generation, six balls were fed (through openings in the top of the machine not shown in the figure), to represent the six somatic chromosomes (three pairs). If the balls are given a distinct color or stamp for each of these eight ancestors, the tracing of the individual balls becomes clearer. The slots are constructed so that the balls, when fed into them, will settle alternately to the right and left, or vice versa, depending upon the chance settling of the first ball. The dividing wedge below the slot is located with its apex on the mid-line of the slot, so that when the slide is withdrawn from the back the balls pass alternately to the right and left, or vice versa, depending upon the particular system of stacking. Thus in the operation of the machine the segregation and recombination of balls follows the same mathematical rules which (barring crossing over, non-disjunction, mutation, special systems of selection, and other special phenomena) govern the descent of chromosomes in normal bisexual heredity. The fundamental formulae given in the accompanying paper may be demonstrated by its use.

This machine is 33 inches by $35^{\frac{1}{2}}$ inches in size, not including the base. Its face is covered with glass $\frac{3}{16}$ inch thick. The dark line traces ancestral descent for the $F_{1}$ zygote. At the bottom of each slot there is a wooden stop which withdraws from the back, and below each cul-de-sac slot there is a tin chute which opens to the rear, and enables the operator conveniently to remove the balls. The three lowest slots also open into similar chutes. 
Assuming the above conditions (and assuming that, until future research results in weighting ontogenetic values of specific chromosomes, each chromosome carries about the same number of genes or determiners for traits as each of the other II chromosomes), we find that we should speak of "blood" or ancestral influence not alone in terms of quotients obtained by dividing unity by the number of direct ancestors of the particular generation, although this has a certain genealogical value, but should measure ancestral influence by the probability that a given number of chromosomes or gene-radicals, according to the demonstrated laws of heredity, will descend from the given ancestors to the particular propositus.

Under the old scheme, each one of the 1.6 great-great-grandparents was thought of as contributing one-sixteenth of the "blood" possessed by the descendants of the $F_{1}$ generation. The average contribution in chromosome-number, (barring the sex-chromosome, which follows much more restricted paths in the family-tree), characteristic of such an ancestor, is I.375. This, of course, is one-sixteenth of the eleven equivalent chromosome-pairs. But this is not a constant contribution. The range of chromosome-contribution by members of the great-great-grandparental generation, barring those individuals in the $\mathrm{X}$ - or $\mathrm{Y}$-trails, varies from none to eleven chromosomes. Each particular case must; of course, be analyzed by pedigree-methods to determine what the actual contribution has been in the case of an individual already born. Actually, (barring special phenomena), each parent or ancestor contributes either none or a definite unit number of chromosomes. This number may be as high as 12 in man, or in any species as high as the gametic number of chromosomes. A fractional number following formula $A$ on the pedigreecharts (plates I and 2) measures an average calculated in reference to the person, taken as a type, occupying the given position in the pedigree.

It is evident that if we wish a more accurate measure of ancestral influence than is given by the ordinary methods, not only must the average number of chromosomes contributed by a given ancestor, taken as a type, be calculated, but also the possible range in number of chromosomes contributed must be stated.

But besides those worked out on the accompanying pedigree-charts, there are many other mathematically measurable features of ancestral influence. For instance, the probability of transmitting from a given ancestor to the $F_{1}$ zygote not only a definite number but a definite combination of specific chromosomes, also the probability of paternity or maternity of descent of particular chromosomes to the ancestor who passes them on, can be formulated. 
Ultimately the biological analysis of a human pedigree will resolve itself into tracing specific chromosomes and parts of chromosomes as the physical determiners of traits, from the propositus back through the familytree. Thus if, as seems probable, there are but is chromosomes in each human gamete, then each half-pedigree, regardless of how many individuals there are in the ancestral generation, can contribute but I 2 chromosomes. For example, in the paternal and maternal half-pedigrees, in the $P_{5}$ generation there are, in each, sixteen direct ancestors; then (barring crossing-over) at least four of these ancestors must be left out entirely so far as chromosomal contribution, and consequently ancestral influence, is concerned. Of course each ancestor is physiologically necessary as a nurse for the germ-plasm, even though in the course of descent all of the chromosomes of a particular ancestor be shunted in the processes of gametogenesis. The location in the pedigree, of the ancestor supplying the gamete which presents a crossing-over in one of the chromosomes, may be established when the knowledge of linkage and the linear geography of chromosomes becomes more exact. Thus until chromosomes are actually weighted as to their relative ontogenetic influence, we must divide "blood" not into halves, quarters, eighths, sixteenths and so on, by the usual genealogical method, but into twenty-four uneven and individual parts. While it is thus proper for genealogical purposes to speak of an individual as fifteen-sixteenths white and one-sixteenth negro, to be biologically correct reference should be to an individual carrying so many (and which) of the total of twenty-four chromosomes from white ancestors and so many from black. It is perfectly proper to speak of a human individual as seven twenty-fourths or nineteen twenty-fourths of one race, provided the twenty-four units are conceived of as individual and, possibly, very unequal elements. With the weighting of chromosomes and the evaluation of fractional chromosomes in crossing-over, the fractions measuring "blood" or ancestral influence will possess no standard denominators, unless perchance such denominator be the ultimate number of human genes.

In any particular zygote there is of course no chance that a given ancestor will contribute anything except what he has actually contributed. The ratios shown on the accompanying pedigree-charts are measures of probability in reference to events which have not yet happened, that is, measures applicable to descendants not yet born. The averages refer not to specific individuals, but to the occupant of a definite position in the pedigree, taken as a type. The ratio of an accompanying formula may be viewed also in the light of an even or a safe bet or probability, 
if we be ignorant of the facts in a specific case, that a given ancestor contributed thus and so from his germ-plasm to the given zygote.

Biologically the formulae here given are as sound as current biological knowledge. They may be modified and developed in accordance with the advance of biological learning. They are equally as applicable to any normally bisexual species of plant or animal as to man.

Mathematically the accompanying formulae are built upon the following elementary principles of (a) probability, and (b) combination.

\section{(a) Probability}

I. The certainty that an event will occur is measured by the quotient $\mathbf{I}: \mathbf{I}$.

2. The impossibility of an event occurring is measured by the quotients $0: \infty$ or $\mathrm{I}: \infty$.

3. If out of $n$ possible happenings, there are no conditions favoring the happening of one possibility over another, the probability that a given event. will occur is $\mathrm{I}: n$.

4. If out of $n$ possible happenings, there are no conditions favoring the happening of one possibility over another, the probability that a given event will not occur is $\mathrm{I}: \frac{n}{n-\mathrm{I}}$.

5. The probability that two or more events will occur is measured by the product of their respective probability-quotients, provided that between or among the several possibilities there is no definite dependence or interference.

6. The probability that none of two or more independent events will occur is measured by the product of the quotients of probability that each event separately will not occur.

7. The probability that a given complex (of certain independent events happening and certain other independent events not happening) will eventuate, is measured by the continued product of the several quotients of probability that each particular constituent event as chosen will or will not occur.

8. The probability that a single possible event will occur within a given portion of a definite range of possibilities is measured by the sum of the probability-quotients of the separate chances constituting the particular given portion.

9. The probability that a single possible event will not occur within a given portion of a definite range of possibilities is $\mathrm{x}: \frac{n}{n-h}$, in which 
$n=$ the total number of equal chances in the range.

$h=$ the number of equal chances in the given portion.

\section{(b) Combinations}

$c=$ whole number of combinations possible within $n$ factors taken $m$ at a time.

$$
c=\frac{n(n-\mathrm{I})(n-2) \ldots(n-m+\mathrm{I})}{\mathrm{I} \cdot 2 \cdot 3 \ldots m} .
$$

$n=$ whole number of factors.

$m=$ number of factors taken at a time (never greater than $n$ ).

FORMULAE FOR CALCULATING ANCESTRAL INFLUENCE IN BISEXUAL SPECIES

The values of formulae number $\mathrm{I}, 4,5,6,7,8,9$ and Io for each individual to whom the particular formulae apply are given on the accompanying charts showing the ancestral influence in the human male, and in the human female. (Plates I and 2.)

I. $A=$ average number of chromosomes passed on by the selected ancestor, taken as a type, to the $\mathbf{F}_{1}$ zygote, i.e., the propositus. $A=$ also the average number of chromosome-pairs each of which contributes one member of the diploid or somatic group of chromosomes of the $\mathbf{F}_{1}$ zygote.

In man the diploid or somatic number of chromosomes is taken tentatively (see explanatory note, page 436 ) at 24 , i.e. Ir equivalent pairs and one $\mathrm{XX}$ or $\mathrm{XY}$ sex pair.

$A=\frac{n}{2^{(p-1)}}+\left(C_{y}\right.$ or $C_{x}$ in case of ancestors from whom an $\mathrm{X}$ or a

$\mathrm{Y}$ chromosome may descend.)

See plates $\mathrm{I}$ and 2 for $\mathrm{X}$ - and $\mathrm{Y}$-trails.

2. $n=$ number of equivalent chromosome-pairs (i.e., excluding the $\mathrm{XY}$ or $\mathrm{XX}$ sex-pair) characteristic of the species. In the case of man $n$ is taken as II.

3. $p=$ the numerical value of the sub-figure of $\mathrm{P}$ used in pedigree-manner to indicate the ancestral or parental generation in relation to the propositus or principal figure of the pedigree. Thus in the great-grandparental generation, $\mathrm{P}_{\hat{\mathrm{s}}}, p=3$.

4. $\quad C_{y}=$ the average number of $\mathrm{Y}$-chromosomes contributed to the $\mathrm{F}_{1}$ zygote by the particular male ancestor taken as a type. 
I $: C_{y}=$ the probability that a particular male ancestor will contribute the single $\mathrm{Y}$-chromosome found in the $\mathrm{F}_{1}$ male zygote.

The value of $\mathrm{I}: C_{y}$ is always $\mathrm{r}: \mathrm{r}$, and it is applicable to the direct male line only. For an ancestor not in the $\mathrm{Y}$-trail, $\mathrm{I}: C_{y}=\mathrm{I}: \propto$.

This direct paternal line down which the $Y$-chromosome descends is the so-called "Y-trail."

5. $C_{x}=$ the average number of $\mathrm{X}$-chromosomes contributed to the $F_{1}$ zygote by the particular ancestor taken as a type.

$\mathrm{I}: C_{x}=$ the probability that the particular male or female ancestor will contribute an $\mathrm{X}$-chromosome to the $\mathrm{F}_{1}$ zygote.

The value of $C_{x}$ is determined by the following rule:

(a) Beginning with the $\mathrm{X}$-chromosomes in the $\mathrm{F}_{1}$ zygote, without exception (i.e., with the chance of $\mathrm{r}: \mathrm{r}$ ) the single $\mathrm{X}$-chromosome in the male is derived from his mother; and of the two $\mathrm{X}$-chromosomes in the female, one is derived from each parent.

(b) In the $P_{1}$ and all older generations, in the case of a female zygote the chance of the descent of an X-chromosome from the father or mother is equal (i.e., I:2), but in the male the $\mathrm{X}$-chromosome is always received from the mother alone (i.e., the chance is $\mathrm{I}: \mathrm{I}$ ).

Therefore, the probability that a given ancestor, taken as a type, will contribute an $\mathrm{X}$-chromosome to the $\mathrm{F}_{1}$ zygote, (i.e., $\mathrm{r}: C_{x}$ ), is equal to the product of all probability ratios, made according to rules (a) and (b) above, along the X-trail from the selected ancestor to the $P_{1}$ generation inclusive.

Thus $\mathrm{r}: C_{x}$ for the mother's mother's father of a female zygote $=(\mathrm{I}: \mathrm{I}) .(\mathrm{I}: 2) .(\mathrm{I}: 2)=\mathrm{I}: 4$; but for the mother's father's mother of a female zygote $\mathrm{I}: C_{x}=(\mathrm{I}: \mathrm{I}) \cdot(\mathrm{I}: 2) .(\mathrm{I}: \mathrm{I})$ $=\mathrm{I}: 2$.

For an ancestor not in the $\mathrm{X}$-trail, $1: C_{x}=\mathrm{I}: \propto$.

Thus, the so-called "X-trail"-from a son to the mother only, but from a daughter to both the father and mother, traces the several lines in the ancestral pedigree down one of which an $\mathrm{X}$-chromosome must descend.

Note: The X-trail and the $\mathrm{Y}$-trail and all calculations herein based upon them apply only to those species in which the male is the heterozygous and the female the homozygous sex. In species in which the reverse is true the $\mathrm{X}$ - and the $\mathrm{Y}$-trails exchange positions. The general rule is that the $\mathrm{Y}$-trail follows the heterozygous sex; the $\mathrm{X}$-trail from the homozygous individual to both parents, but from a heterozygous individual to the homozygous parent only. 
6. $R$ = range in number of chromosomes (i.e., in number of single members of chromosome-pairs) possible for the selected ancestor, taken as a type, to contribute to the 24 (i.e., I I equivalent pairs and I XY or I XX pair) chromosomes of the $F_{1}$ zygote.

In a parent of the $P_{1}$ generation, $R=\mathrm{I}_{2} \rightarrow \mathrm{I} 2$, i.e., there is no range in the number of chromosomes which the given parent may contribute to the $F_{1}$ zygote; this number is always $I 2$. For all ancestors of the $\mathrm{P}_{2}$ and all older generations, $R=0 \rightarrow \mathrm{II}$, except (a) for those ancestors from whom an X- or a $\mathrm{Y}$ chromosome must certainly descend (solid line X-trail or Y-trail on chart), in whom $R=\mathrm{I} \rightarrow \mathrm{I} 2$; except also (b) for those ancestors from whom an X-chromosome may, by alternative chance, descend (dotted line $\mathrm{X}$-trail in chart), in whom $R=\mathrm{o} \rightarrow \mathrm{I} 2$.

In a general formula i 1 would be replaced by $n$, and 2 by $n+\mathrm{I}$, with the value of $n$ as in formula 2 .

7. $\mathrm{I}: C_{t}=$ the probability (barring mutation, crossing over and other special phenomena) that one or the other member of each of all of the equivalent chromosome-pairs characteristic of the species (i.e., an entire chromosome-series excluding the $\mathrm{XX}$ and $\mathrm{XY}$ pairs), from the given ancestor will enter the $F_{1}$ zygote.

In the case of ancestors not in the $\mathrm{X}$-trail or the $\mathrm{Y}$-trail, such an occurrence is the extreme limit of ancestral influence possible to a given ancestor, and its happening precludes any hereditary influence at all on the propositus, i.e., the $F_{1} z y-$ gote, on the part of any other ancestor of the same paternal or maternal half-pedigree, as the case may be, except the lineal ancestors and descendants of the given ancestor; consequently $\mathrm{I}: C_{t}$ also measures the probability that the selected ancestor will contribute all of the representatives of the equivalent chromosome-pairs entering the $F_{1}$ zygote from the particular halfpedigree and generation.

$\mathrm{I}: C_{t}=\mathrm{I}:\left(2^{(p-1)}\right)^{n}$

$n=$ value as in formula 2. In man $n$ is taken as $\mathbf{I} \mathbf{I}$.

$p=$ value as in formula 3 .

Note: Formula 7 is in fact a special case of formula 17. 
8. $\mathrm{I}: C_{t x}$ or $\mathrm{I}: C_{t y}=$ the probability (barring mutation, crossing over and other special phenomena) that one or the other member of each of the several equivalent chromosome-pairs characteristic of the species, and also one $\mathrm{X}$ - or one Y-chromosome, as the case may be, from the given ancestor will enter the $F_{1}$ zygote. (In man this total gametic number of chromosomes is taken as 12 .)

Such an occurrence can happen only in reference to ancestors in the $\mathrm{X}$ - or Y-trails, and its happening precludes any hereditary influence at all on the propositus, i.e., the $F_{1} z y-$ gote, on the part of any other ancestor of the same paternal or maternal half-pedigree, except the lineal ancestors and descendants of the given ancestor.

$$
\mathbf{\mathbf { r }}: C_{t x}=\left(\mathbf{\mathbf { r }}: C_{t}\right) \cdot\left(\mathbf{\mathbf { r }}: C_{x}\right)
$$

Values of $\mathrm{I}: C_{t}$ and $\mathrm{I}: C_{x}$ as in formulas 5 and 7 . $\mathrm{I}: C_{t y}=\left(\mathrm{I}: C_{t}\right) \cdot\left(\mathrm{I}: C_{y}\right)$

Values of $\mathrm{r}: C_{t}$ and $\mathrm{r}: C_{y}$ as in formulas 4 and 7 .

9. $\mathrm{I}: C_{-t}=$ the probability (barring mutation, crossing over and other special phenomena) that no member of any of the equivalent chromosome-pairs characteristic of the species (thus barring for the time being all consideration of the $\mathrm{XY}$ or XX chromosome-pairs) from the given ancestor will enter the $F_{1}$ zygote.

In such an occurrence the given ancestor and all of his lineal ancestors are entirely eliminated as contributors to the natural inheritance of the propositus, i.e., the $F_{1}$ zygote, provided that such given ancestor is not in the X-trail or $\mathrm{Y}$ trail, and does not, due to inbreeding, appear elsewhere in the ancestral pedigree.

$$
\mathrm{I}: C_{-i}=\mathrm{I}:\left(\frac{2^{(p-1)}}{2^{(p-1)}-\mathrm{I}}\right)^{n}
$$

$n=$ value as in formula 2 .

$p=$ value as in formula 3 .

$\mathrm{I}: C_{-t}$ measures the probability that a selected ancestor will contribute none of the representatives of the equivalent chromosome-pairs entering the $F_{1}$ zygote from the particular half-pedigree.

Therefore, the probability that the selected ancestor will contribute all of the representatives of the equivalent chromosome-pairs equals the probability that all of the remaining members of the same ancestral generation of the same half- 
pedigree will be left out entirely as contributors of members of equivalent chromosome pairs to the $F_{1}$ zygote; whence the check for formula 7 ,

$$
\mathrm{I}: C_{t}=\mathrm{I}:\left(C_{-t}\right) \cdot\left(2^{(p-1)}-\mathrm{I}\right)^{n}
$$

in which $2^{(p-1)}-I$ is always the number of ancestors of the particular generation of the same half-pedigree who contribute nothing in case the one remaining member contributes all, and in which $n$ equals the number of equivalent chromosome-pairs characteristic of the species.

Still more generally the probability that a given combination of members of the same ancestral generation of the same half-pedigree will contribute no representative of the equivalent chromosome-pairs to the $F_{1}$ zygote equals $x:\left(C_{-t}\right) \cdot m^{n}$ in which $m$ is the number of members of the selected combination of ancestors of the same generation and the same half-pedigree which cannot exceed $2^{(p-1)}-\mathrm{I}$.

Note; It is apparent that the probability that a given ancestor will contribute no member of any equivalent chromosome pairs to the $F_{1}$ zygote (formula 9), is quite different from the probability that a given ancestor will not contribute all of the chromosome representative of the entire chromosomal series. The latter is the negative of formula 7 , which negative is stated, $\mathrm{I}: \frac{\left(2^{(p-1)}\right)^{n}}{\left(2^{\left.\left({ }^{p-1}\right)\right)^{n}-\mathrm{I}}\right.}$

Note: Formula 9 is in fact a special case under formula 19.

Iо. $\mathrm{I}: C_{-t x}$ or $\mathrm{I}: C_{-t y}=$ the probability (barring mutation, crossing over and other special phenomena) that no member of any equivalent chromosome-pair, nor of the XX or XY chromosomepair, from the given ancestor will enter the $F_{1}$ zygote, i.e., the probability (barring crossing over and other special phenomena, and the appearance of the given ancestor elsewhere in the pedigree) of the complete elimination of the hereditary influence of the given ancestor and all of his or her lineal ancestors, on the propositus, i.e., the $\mathrm{F}_{1}$ zygote.

This formula is applicable only to those ancestors in the $X$ trail or $Y$-trail.

$$
\mathrm{I}: C_{-t x}=\left(\mathrm{I}: C_{-t}\right) \cdot\left(\mathrm{r}: C_{-x}\right)
$$

Values of $\mathrm{I}: C_{-}$and $\mathrm{I}: C_{-x}$ as in formula 9 above and $\mathrm{II}$ following.

$$
\begin{aligned}
& \mathrm{r}: C_{-t y}=\left(\mathrm{r}: C_{-t}\right) \cdot\left(\mathrm{r}: C_{-u}\right) \\
& \text { Values of } \mathrm{I}: C_{-t} \text { and } \mathrm{I}: C_{-y} \text { as in formula } 9 \text { above and } \mathrm{II} \\
& \text { following. }
\end{aligned}
$$


II. I: $C_{-x}=$ the probability that a given ancestor who is in the $\mathrm{X}$-trail will not contribute an $\mathrm{X}$-chromosome to the $\mathrm{F}_{1}$ zygote.

$$
\mathrm{I}: C_{-x}=\mathrm{I}: \frac{C_{x}}{C_{x}-\mathrm{I}}
$$

For an ancestor not in the $\mathrm{X}$-trail, $\mathrm{I}: C_{-x}=\mathrm{I}: \mathrm{I}$.

Value of $C_{x}$ as in formula 5 .

I2. I: $C_{-y}=$ the probability that a given ancestor who is in the $\mathrm{Y}$-trail will not contribute a $\mathrm{Y}$-chromosome to the $\mathrm{F}_{1}$ zygote.

$$
\mathrm{I}: C_{-y}=\mathrm{I}: \frac{C_{y}}{C_{y}-\mathrm{I}}
$$

For an ancestor not in the $\mathrm{Y}$-trail, $\mathrm{I}: C_{-y}=\mathrm{I}: \mathrm{I}$.

Value of $C_{y}$ as in formula 4 .

I3. I: $G_{a}=$ the probability that one or the other member of an allelomorphic pair of Mendelian genes (disregarding genes in the $\mathrm{XX}$ or XY sex-chromosomes), regardless of whether the selected member is dominant or recessive to its allelomorph, from the given ancestor will enter the $F_{1}$ zygote.

$\mathrm{I}: G_{a}=\mathrm{I}: 2(\underline{p}-1)$

$G_{a}=2^{(p-1)}=$ the number of ancestors in a given half-generation. I: $G_{a}=$ also the probability that the entire chromosome, (barring crossing over, non-disjunction, and other special phenomena), of which the particular gene is a part will enter the $F_{1}$ zygote.

$p=$ value as in formula 3 .

NotE: This ratio may well be called the foundation formula of human heredity. As biological knowledge advances other subsidiary formulae may be deduced and synthesized with it.

I4. $\mathrm{r}: G_{-a}=$ the probability that neither member of a given allelomorphic pair of Mendelian genes (disregarding genes in the XX or XY sex-chromosomes) from the given ancestor will enter the $F_{1}$ zygote.

$$
\mathrm{I}: G_{-a}=\mathrm{I}: \frac{\mathrm{I}}{\mathrm{I}-\frac{\mathrm{I}}{2^{(p-1)}}}
$$

$p=$ value as in formula 3 .

$\mathrm{I}: G_{-a}=$ also the probability that neither member of the entire chromosome-pair, barring crossing over, non-disjunction and other special phenomena, of which the particular gene is a part, will enter the $F_{1}$ zygote.

I5. $\mathrm{I}: G_{b}=$ the probability that a definitely selected paternally or maternally contributed member of an allelomorphic pair of Mendelian genes (disregarding genes in the XX or XY sex-chromosomes), 
regardless of whether the particular member is dominant or recessive to its allelomorph, from the given ancestor will enter the $F_{1}$ zygote.

$\mathrm{I}: G_{b}=\mathrm{I}: 2^{p}$

$\mathrm{I}: G_{b}=$ also the probability that, barring crossing-over, non-disjunction and other special phenomena, the entire chromosome, of which the particular gene is a part will enter the $F_{1}$ zygote. $p=$ value as in formula 3 .

I6. $I: G_{-b}=$ the probability that a definitely selected paternally or maternally contributed member of a pair of Mendelian genes, (disregarding genes in the $\mathrm{XX}$ or $\mathrm{XY}$ sex-chromosomes), from the given ancestor will not enter the $\mathrm{F}_{1}$ zygote.

$$
\mathrm{I}: G_{-b}=\mathrm{I}: \frac{\mathrm{I}}{\mathrm{I}-\frac{\mathrm{I}}{2^{p}}}
$$

$p=$ value as in formula 3 .

I $: G_{-b}=$ also the probability that, barring crossing over, non-disjunction and other special phenomena, the entire chromosome of which the particular gene is a part will not enter the $\mathbf{F}_{1}$ zygote.

I7. $\mathrm{I}: C_{a}=$ the probability (barring mutation, crossing over and other special phenomena) that one or the other member from each of a definitely selected combination of equivalent chromosome-pairs (i.e., a definite combination among the chromosomes $a$ to $k$, if I I be the number of such equivalent pairs in man, thus disregarding the alternative $\mathrm{XX}$ and $\mathrm{XY}$ sex-pairs), from the given ancestor, regardless of the behavior of other chromosome-pairs, will enter the $F_{1}$ zygote.

$\mathrm{I}: C_{a}=\mathrm{I}:\left(2^{(p-1))^{a}}\right.$

$p=$ value as in formula 3 .

$a=$ the number of such chromosomes in the definitely selected combination, (which in man cannot exceed II).

$\mathbf{I}:\left(2^{p-1}\right)^{a}=$ also the probability that a definite combination of one of the other allelomorphs of non-linked genes, barring crossing over, non-disjunction and other special phenomena, carried by the given ancestor, will enter the $F_{1}$ zygote.

18. I $: C_{b}=$ the probability (barring mutation, crossing over, and other special phenomena) that a definitely selected paternally or maternally contributed member from each of a definitely selected combination of equivalent chromosome-pairs (i.e., a definite combination among chromosomes $a$ to $k$, if I $\mathrm{I}$ be the number 
of such equivalent pairs in man, thus disregarding the alternative $\mathrm{XX}$ and $\mathrm{XY}$ sex-pairs) from the given ancestor, regardless of the behavior of other chromosome-pairs, will enter the $F_{1}$ zygote.

$\mathrm{I}: C_{b}=\mathrm{I}:\left(2^{p}\right)^{b}$

$p=$ value as in formula 3 .

$b=$ number of definitely selected paternally or maternally descended chromosomes in the definite combination passed on by the given ancestor.

$\mathrm{I}:\left(2^{p}\right)^{b}=$ also the probability that a definite combination of definitely selected paternally or maternally descended non-linked genes, barring crossing over, non-disjunction and other special phenomena, carried by the given ancestor will enter the $F_{1}$ zygote.

I9. I: $C_{-c}=$ the probability that neither member of any pair of a definitely selected combination of equivalent chromosome-pairs (i.e., a definite combination among chromosome-pairs $a$ to $k$, if $\mathrm{I}$ I be the correct number in man, thus disregarding the alternative $\mathrm{XX}$ and $\mathrm{XY}$ sex-pairs) from the given ancestor, regardless of the behavior of other chromosome-pairs, will enter the $F_{1}$ zygote.

$$
\mathrm{I}: C_{-c}=\mathrm{I}: \frac{\mathrm{I}}{\left(\mathrm{I}-\frac{\mathrm{I}}{2^{(p-1)}}\right)^{c}}
$$

$c=$ number of chromosomes in the definitely selected combination barred from the $F_{1}$ zygote. In man it must not be greater than Ir.

$p=$ value as in formula 3 .

I: $C_{-c}=$ also the probability that neither allelomorph of a definitely selected combination of non-linked Mendelian genes, barring crossing over, non-disjunction and other special phenomena, carried by the given ancestor will enter the $F_{1}$ zygote.

20. $\mathrm{I}: C_{d}=$ the probability (barring mutation, crossing-over and other special phenomena) that one or the other member of a definitely selected combination of equivalent chromosome-pairs (definite combinations among the chromosome-pairs $a$ to $k$, if II be the correct number of equivalent chromosome-pairs in man, thus disregarding the alternative $\mathrm{XX}$ and $\mathrm{XY}$ sex-pair), and no more chromosomes from the given ancestor will enter the $F_{1}$ zygote. 


$$
\begin{aligned}
\mathrm{I}: C_{d} & =\mathrm{I}: \frac{\left(2^{(p-1)) a}\right.}{\left(\mathrm{I}-\frac{\mathrm{I}}{2^{(p-1)}}\right)^{c}} \\
a & =\text { value as in formula } \mathbf{I} 7 . \\
n & =\text { value as in formula } 2 . \\
p & =\text { value as in formula } 3 . \\
c & =\text { value as in formula } \mathrm{I} 9 .
\end{aligned}
$$

NoTE: This formula is the product of formula if times formula 19 , in which always the number of chromosomes entering plus the number barred from the $\mathrm{F}_{1}$ zygote $=n$, i.e., $a+c=n$.

2I. I: $C_{e}=$ the probability (barring mutation, crossing over and other special phenomena) that a definitely selected paternally or maternally contributed member from each of a definitely selected combination of equivalent chromosome-pairs (i.e., a definite combination among chromosome-pairs $a$ to $k$, if I I be the number of such equivalent pairs in man, thus disregarding the alternative $\mathrm{XX}$ and $\mathrm{XY}$ sex-pairs), and no more chromosomes, from the given ancestor, will enter the $F_{1}$ zygote.

$$
\begin{aligned}
\mathrm{I}: C_{e} & =\mathrm{I}: \frac{\left(2^{p}\right)^{b}}{\left(\mathrm{I}-\frac{\mathrm{I}}{2^{(p-1)}}\right)^{c}} \\
b & =\text { value as in formula } \mathrm{I} 8 . \\
n & =\text { value as in formula } 2 . \\
p & =\text { value as in formula } 3 . \\
c & =\text { value as in formula } \mathrm{I} g .
\end{aligned}
$$

NOTE: This formula is the product of formula $\mathrm{I} 8$ times formula $\mathrm{I} 9$, in which always the number of chromosomes entering plus the number barred from the $\mathrm{F}_{1}$ zygote $=n$, i.e., $a+c=n$.

22. I: $N_{a}=$ the probability (barring mutation, crossing over, and other special phenomena) that a definite number (but no more) of chromosomes from the given ancestor, regardless of their individuality, and of their paternal or maternal descent, will enter the $F_{1}$ zygote.

$$
\mathrm{I}: N_{\mathrm{a}}=\mathrm{I}: \frac{\frac{\left(2^{(p-1)}\right)^{d}}{\left(\mathrm{I}-\frac{\mathrm{I}}{2^{(p-1)}}\right)^{c}}}{\frac{n \cdot(n-\mathrm{I}) \cdot(n-2) \cdot(n-3) \ldots(n-d+\mathrm{I})}{\mathrm{I} \cdot 2 \cdot 3 \ldots d}}
$$


$d=$ the definite number of chromosomes (regardless of combinations) passed on to the $F_{1}$ zygote.

$n=$ value as in formula 2 .

$p=$ value as in formula 3 .

$c=n-d$.

23. I: $N_{b}=$ the probability (barring mutation, crossing over and other special phenomena) that a definite number (but no more) of chromosomes from the given ancestor, regardless of their individuality, but specified in total numbers as to the maternity and paternity of their descent, will enter the $F_{I}$ zygote.

$\mathrm{I}: N_{b}=\mathrm{I}: \frac{\frac{\left(2^{(p-1)}\right)^{d} \cdot 2^{d}}{\left(\mathrm{I}-\frac{\mathrm{I}}{\left(2^{(p-1)}\right)}\right)^{c}}}{\frac{n \cdot(n-\mathrm{I}) \cdot(n-2) \cdot \ldots(n-d+\mathrm{I})}{\mathrm{I} \cdot 2 \cdot 3 \ldots d} \times \frac{d \cdot(d-\mathrm{I}) \cdot(d-2) \ldots(d-s+\mathrm{I})}{\mathrm{I} \cdot 2 \cdot 3 \ldots . s}}$

$d=$ value as in formula 22 .

$n=$ value as in formula 2 .

$p=$ value as in formula 3 .

$c=n-d$.

$s=$ the number of chromosomes originating in the selected parent of the given ancestor, that will enter the $F_{1}$ zygote.

$d-s=$ the number of chromosomes originating in the other parent of the given ancestor, that will enter the $F_{1}$ zygote.

24. In case of inbreeding or consanguineous matings, the measure of ancestral influence (barring mutation, crossing over, and other special phenomena) is determined as follows:

(a) $A_{m}=$ the average number of chromosomes which a given ancestor, taken as a type, who appears more than once in the direct ancestry, contributes to the propositus.

$A_{m}=$ the sum of the several values of $A$ for each of the several appearances of the particular ancestor in the pedigree.

(b) $R_{m}=$ the range in possible number of chromosomes which a given ancestor, taken as a type, who appears more than once in the direct ancestry, contributes to the propositus.

$$
R_{m}=r^{\prime} \rightarrow r^{\prime \prime} \text { (i.e., from } r^{\prime} \text { to } r^{\prime \prime} \text { ). }
$$

$r^{\prime}=$ the highest of the several minima in the several values of $R$ for the given ancestor in his or her several places in the direct ancestry. 
$r^{\prime \prime}=$ the highest of the several maxima in the several values of $R$ for the given ancestor in his or her several places in the direct ancestry.

(c) I : $C_{t m x}=$ the probability that a given ancestor, taken as a type, who appears more than once in the direct ancestry, will contribute from one or more of the several such appearances all of the chromosomes supplied by the particular halfpedigree to the propositus.

$$
\begin{aligned}
\mathrm{I}: C_{t m x}=\mathrm{I}: \frac{\left(\begin{array}{l}
h \\
g
\end{array}\right)^{n}}{\left(\mathrm{I}: C_{x}\right) \cdot\left(\mathrm{I}: C_{y}\right)} \\
n=\text { value as in formula } 2 . \\
h=\text { the total number of direct ancestors in the } \\
\quad \text { oldest half-generation in which the particu- } \\
\quad \text { lar inbred ancestor appears }(=2(p-1)) . \\
p=\text { value as in formula } 3 . \\
g=\text { the number of parts of the particular half-pedi- } \\
\quad \text { gree covered by the given ancestor, in terms } \\
\quad \text { of } h \text { units. } \\
C_{x}=\text { value as in formula } 4 . \\
C_{y}=\text { value as in formula } 5 . \\
\text { (d) } \mathrm{I}: C_{-t m x}=\text { the probability that a given ancestor, taken }
\end{aligned}
$$
as a type, who appears more than once in the direct ancestry will contribute no chromosomes to the propositus.

$$
\begin{aligned}
& \text { I: } C_{-t m x}=\mathrm{I}:\left(\frac{\frac{h}{g}}{\frac{h}{g}-\mathrm{I}}\right)^{n} \cdot\left(C_{-x a} \cdot C_{-x b} \cdots\right) \cdot\left(C_{-y}\right) \\
& n=\text { value as in formula } 2 . \\
& h=\text { value as in formula } 24(\mathrm{c}) . \\
& g=\text { value as in formula } 24(\mathrm{c}) . \\
& C_{-x}=\text { value as in formula I } \mathrm{I} . \quad(a, b, \text { and so on, follow- } \\
& \quad \text { ing the } x \text { in the formula, refers to } C_{-x} \text { in the } \\
& \quad \text { first, second and other appearances of the pro- } \\
& \quad \text { positus in the X-trail }) .
\end{aligned}
$$

It is obvious that 24 (c) or 24 (d), if in the given case the last two parentheses be omitted, measures the given probability in reference to all of the chromosomes except the $\mathrm{X}$ and Y sex-members. Also if $n$ represent a definite combination of $n$ 
specific chromosomes, regardless of the maternity or paternity of their descent and regardless of the behavior of other chromosomes, the formula without the last two parentheses is true for the specific chromosomal combination selected. Also these same formulae without the first parenthesis measure the given probability for the $\mathrm{X}$ and $\mathrm{Y}$ sex-chromosomes.

(e) The probability that the propositus will be a zygotic chromosomal replica of a given ancestor who appears once or more in both the paternal and maternal half-pedigrees is equal to the product of the probability-quotient that the particular inbred ancestor will supply all of the chromosomes contributed to the $F_{1}$ zygote by the paternal half-pedigree (formula 24 (c)) times the same probability-quotient in reference to the maternal half-pedigree times $1: 2^{(n+1)}$.

$n=$ value as in formula 2 .

It is obvious that in the case of species with XY-type of male and XX-type of female a male replica can occur only if the repeating ancestor is a male and appears at least in both the $\mathrm{X}$ and Y-trails; the female replica only if the repeating ancestor is a female and appears at least in the X-trail in both the paternal and maternal sides of the house. The formula, however, takes all of these chances into consideration.

A zygotic chromosomal replica in relation to its repeated ancestor is biologically the equivalent of one identical twin to its mate, although in the case of chromosomal replicas the natural traits have been segregated, distributed among several direct ancestors and later reassembled in the replica, while in the case of identical twins the duplication is immediate from the zygote.

25. $S=$ the minimum number of ancestors in a given ancestral generation among whom will be found all of the possible contributors of the sex-chromosomes (i.e., $\mathrm{XX}$ or $\mathrm{XY}$ ) to the $\mathrm{F}_{1}$ zygote. $p=$ the number of the given ancestral generation: e.g., for parents, $p=\mathrm{I}$; for grandparents, $p=2$; for great-grandparents, $p=$ 3 ; and so on as in formula 3 .

In the human female in which the sex-chromosome formula is $\mathrm{X}+\mathrm{X}, S=$ the $(p+3)$ th member of the Fibonacci series. The maternal half-pedigree provides one $\mathrm{X}$-chromosome in which half-pedigree $S=$ Fibonacci serial term $p+2$. In the paternal half-pedigree which also contributes one $\mathrm{X}$ chromosome, $S=$ Fibonacci serial term $p+\mathrm{r}$. 
In the human male the maternal half-pedigree contributes an X-chromosome, the paternal half-pedigree a $\mathrm{Y}$-chromosome. In this sex for both half-pedigrees united, $S=$ (Fibonacci serial term $p+2)+$ I. In the maternal half pedigree $S=$ Fibonacci serial term $p+2$, in the paternal half-pedigree $S$ $=\mathrm{I}$.

Fibonacci series $=0, \mathrm{I}, \mathrm{I}, 2,3,5,8, \mathrm{I} 3,2 \mathrm{I}, 34,55, \ldots$. , in which each term is the sum of the two preceding terms.

\section{SUMMARY}

I. The present problem of measuring ancestral influence by tracing chromosomes or gene-radicals is demonstrated to be a special case under the following general situation:

Given:

(a) A single biological unit factor (a gene) in a known situation (in a $P_{1}$ ancestor) and the quotient measuring the probability of its reacting in a definite manner or reaching a definite new situation at the end of a single move or transformation (in the $F_{1}$ zygote).

(b) The demonstrated synchronization or sequence of action of two or more such biological unit factors (several genes through several generations).

\section{Solution:}

By applying the correct principles of combination and chance, the probability that a given complex situation will result from a given set of constituent conditions at the end of a given number of definite kinds of transformations is mathematically formulated.

In the case of measuring ancestral influence by tracing chromosomal descent, the situations and transformations concern definite chromosomecomplexes in the zygote and their breaking-up, their recombination and their transmission to offspring in accordance with the principles demonstrated by genetics and cytology.

2. The formulae of bisexual heredity here set forth are foundational. They are stated in general terms whose validity depends upon their presenting correct mathematical pictures of the chromosomal processes which work out in gametogenesis and fertilization.

3. When knowledge of a special case or type of chromosomal behavior is worked out, the mathematical formula which describes the particular case may be joined to the foundational formulae here given, and thus the the general mathematical formula of bisexual heredity may be gradually approximated. 


\section{LITERATURE CITED}

Harvey, Ethel Browne, rgi6 A review of the chromosome numbers in the metazoa. Part I. Jour. Morph., 28: $1-63$.

I920 A review of the chromosome numbers in the metazoa. Part II. Jour. Morph., 34: $\mathrm{I}-67$.

von Winiwarter, H., Igr 2 Etudes sur la spermatogénèse humaine. Arch. d. Biol. $27: 9 \mathrm{I}-\mathrm{r} 89$. WeIMAN, H.L., 1917 The chromosomes of human spermatocytes. Amer. Jour. Anat. 21: I-2 1.

\section{LEGEND FOR $\sigma^{7}$ CHROMOSOME-ORIGIN CHART (PLATE I)}

Notes: The calculations on this chart (a) are based upon the theory that the zygotic chromosome-formula for the human male is $(\mathrm{II}+\mathrm{IX})+(\mathrm{II}+\mathrm{rY}$ ), and (b) do not account for mutation, crossing over, special schemes of selection or other special phenomena.

The formulae upon which these calculations are based are general and foundational, and apply to any bisexual species for which the chromosome-number is given. They may be corrected to account for special phenomena by the articulation of formulae for particular processes when the latter are mathematically generalized.

$A=$ average number of chromosomes passed on by the selected ancestor to the $F_{1}$ zygote.

(Formula I.)

$\Sigma A$ for all of the ancestors of a given generation $=24$.

$R=$ range within the haploid number of chromosomes (i.e., number of single members of chromosome pairs) possible for the selected ancestor to contribute to the 24 (i.e., II equivalent pairs and the $\mathrm{XY}$ sex-pair) chromosomes of the $\mathrm{F}_{1}$ zygote. Barring crossing over each actual contribution is a whole number. (Formula 6.)

$C_{t}=$ the chance that one or the other member of each equivalent chromosome pair, i.e., an entire haploid series, excluding a member of the XY sex-pair, in a given ancestor will enter the $F_{1}$ zygote. (Formula 7 .)

$C_{-t}=$ the chance that no member of any equivalent chromosome pair in a given ancestor will enter the $F_{1}$ zygote. (Formula 9.)

$C_{x}=$ the chance that the selected ancestor carried the single X-chromosome that will enter the $F_{1} 0^{3}$ zygote. (Formula 5.)

Also $=$ the average number of $\mathrm{X}$-chromosomes contributed by the selected ancestor to the $\mathrm{F}_{\mathbf{1}}$ zygote.

$C_{y}=$ the chance that the selected ancestor carried the single Y-chromosome that will enter the $\mathbf{F}_{1} \mathrm{o}^{7}$ zygote. (Formula 4.)

Also $=$ the average number of $Y$-chromosomes contributed by the selected ancestor to the $F_{1}$ zygote.

$C_{t x}$ or $C_{t y}=$ the chance that one or the other member of each equivalent chromosome pair and also one $\mathrm{X}$ - or $\mathrm{Y}$-chromosome in the selected ancestor, will enter the $F_{1}$ zygote, i.e., that the selected ancestor represents all of the ancestral influence of her or his generation in her or his half-pedigree. (Formula 8.)

$C_{-t x}$ or $C_{-t y}=$ the chance that no member of any equivalent chromosome pair nor of the $\mathrm{XX}$ or XY sex-pair in the selected ancestor will enter the $F_{1}$ zygote, i.e., the chance of complete elimination of the ancestral influence of the selected ancestor. (Formula Io.)

For the $F_{1}$ male zygote

Certain selected formula-values for given ancestors in the $P_{4}$ generation

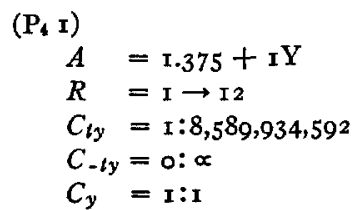




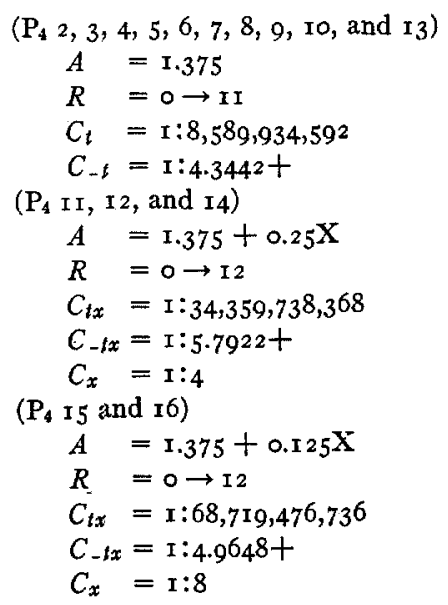

For the $F_{1}$ male zygote

$\left(\mathrm{P}_{\mathbf{b}} \mathrm{I}\right)$

Certain selected formula-values for given ancestors in the $P_{6}$ generation

$$
\begin{aligned}
& A=0.6875+\mathrm{IY} \\
& R=\mathrm{I} \rightarrow \mathrm{I} 2 \\
& C_{t y}=\mathrm{I}: \mathrm{I} 7,592,186,044,4 \mathrm{r} 6 \\
& C_{-t y}=0: \propto \\
& C_{y}=\mathrm{I}: \mathrm{I}
\end{aligned}
$$

( $\mathrm{P}_{6} 2,3,4,5,6,7,8,9, \mathrm{IO}, \mathrm{II}, \mathrm{I2}, \mathrm{I} 3, \mathrm{I4}, \mathrm{I5}, \mathrm{I6}, \mathrm{I7}, \mathrm{I} 8, \mathrm{I9}, 20,2 \mathrm{I}, 25,26$, and 29)

$$
\begin{aligned}
& A=0.6875 \\
& R=0 \rightarrow \mathrm{II} \\
& C_{t}=\mathrm{I}: 17,592,186,044,416 \\
& C_{-t}=\mathrm{I}: 2.0338+
\end{aligned}
$$

$\left(\mathrm{P}_{6}\right.$ 22)

$$
\begin{aligned}
& A=0.6875+0.25 \mathrm{X} \\
& R=0 \rightarrow \mathrm{I} 2 \\
& C_{t x}=\mathrm{I}: 70,368,744,177,664 \\
& C_{-t x}=\mathrm{I}: 2.7 \mathrm{II} 7+ \\
& C_{x}=\mathrm{I}: 4
\end{aligned}
$$

$\left(P_{5} 23,24,27,28\right.$, and 30$)$

$A=0.6875+0.125 \mathrm{X}$

$R \quad=0 \rightarrow 12$

$C t x=\mathrm{I}: \mathrm{I} 4 \mathrm{O}, 737,488,355,328$

$C_{-t x}=1: 2.3243+$

$C x=\mathrm{r}: 8$

( $\mathrm{P}_{5} 3 \mathrm{I}$ and 32)

$$
\begin{aligned}
& A=0.6875+0.0625 \mathrm{X} \\
& R=0 \rightarrow \mathrm{I} 2 \\
& C_{t x}=\mathrm{I}: 28 \mathrm{r}, 474,976,710,656 \\
& C_{-t x}=\mathrm{I}: 2.1693 \\
& C_{x}=\mathrm{I}: \mathrm{I} 6
\end{aligned}
$$




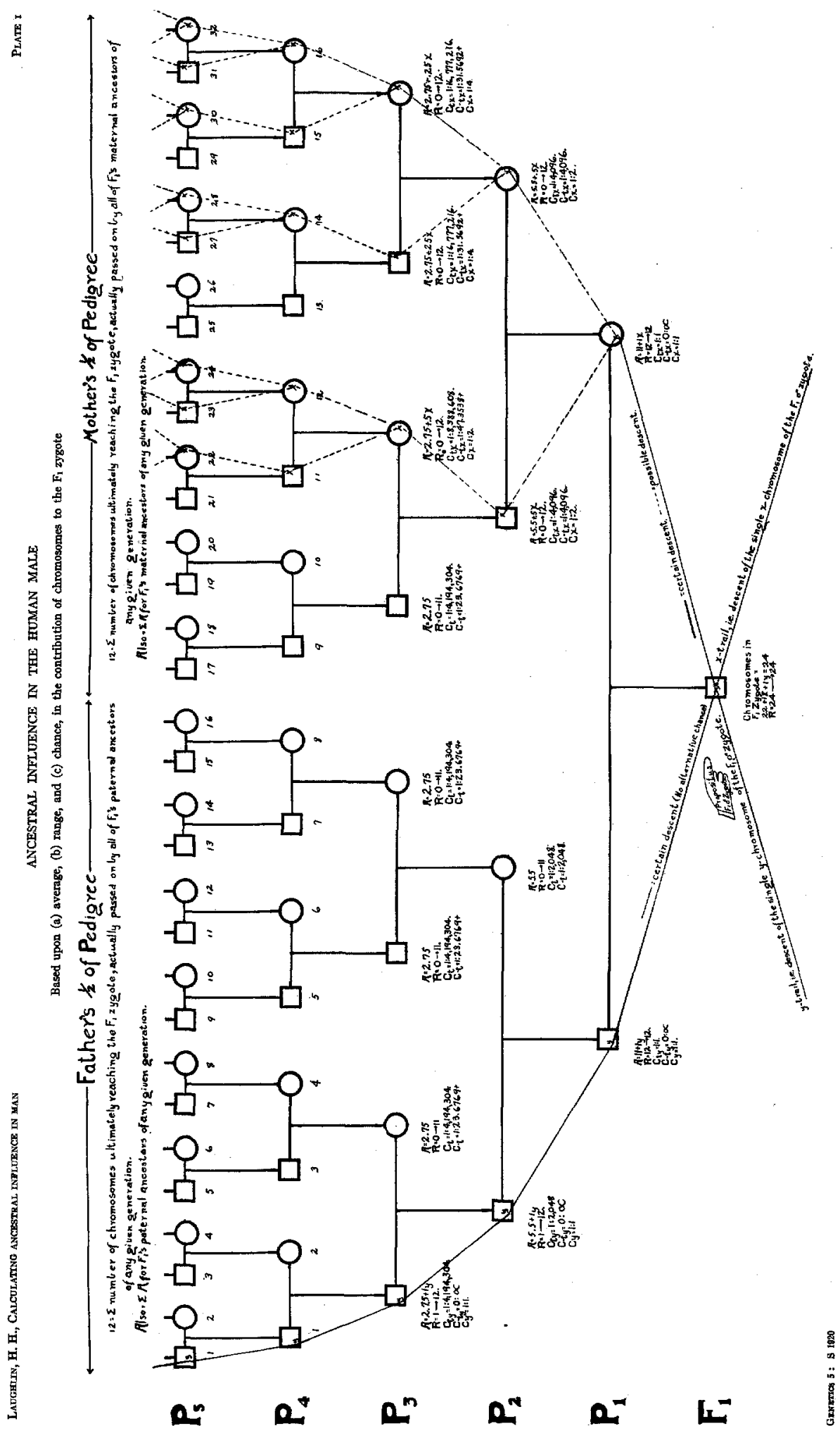




\section{IEGEND FOR $\%$ CHROMOSOME-ORIGIN CHART (PLATE 2)}

NoTE: These calculations (a) are based upon the theory that the zygotic chromosomeformula for the human female is $(\mathrm{I} I+\mathrm{IX})+(\mathrm{II}+\mathrm{IX})$, and (b) do not account for mutation, crossing over, special schemes of selection or other special phenomena.

$A=$ average number of chromosomes passed on by the selected ancestor to the $F_{1}$ zygote. (Formula I.)

$\Sigma A$ for all of the ancestors of a given generation $=24$.

$R=$ range within the haploid number of chromosomes (i.e., the number of single members of chromosome pairs) possible for the selected ancestor to contribute to the 24 (i.e.,

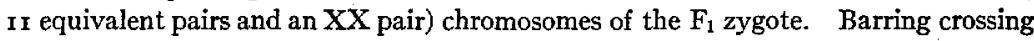
over each actual contribution is a whole number. (Formula 6.)

$C_{t}=$ the chance that one or the other member of each equivalent chromosome-pair (i.e., an entire haploid series excluding the $\mathrm{X}$-chromosome) in a given ancestor will enter the $F_{1}$ zygote. (Formula 7 .)

$C_{-t}=$ the chance that no member of any equivalent chromosome pair, excluding the XY sexpair, in a given ancestor will enter the $F_{1}$ zygote. (Formula 9.)

$C_{x}=$ the chance that the selected ancestor carried one of the X-chromosomes that will enter the $F_{1} \&$ zygote. (Formula 5.)

Also $=$ the average number of $\mathrm{X}$-chromosomes contributed by the selected ancestor to the $\mathrm{F}_{1}$ zygote.

$C t x=$ the chance that one or the other member of each equivalent chromosome-pair and also one $\mathrm{X}$-chromosome in the selected ancestor will enter the $F_{1}$ zygote, i.e., that the selected ancestor represents all of the ancestral influence in her or his generation in her or his half-pedigree. (Formula 8.)

$C . t x=$ the chance that no member of any equivalent chromosome-pair nor of the $\mathrm{XX}$ or $\mathrm{XY}$ sex-pair in the selected ancestor will enter the $F_{1}$ zygote, i.e., the chance of complete elimination of the ancestral influence of the selected ancestor. (Formula ro.)

For the $F_{1}$ female zygote

Certain selected formula-values for given ancestors in the $\mathrm{P}_{\mathbf{4}}$ generation

$$
\begin{aligned}
\left(\mathrm{P}_{4} \mathrm{I}, 2,3,4,5,9,10, \text { and } \mathrm{I} 3\right) & \\
A & =\mathrm{I} .375 \\
R & =0 \rightarrow \mathrm{II} \\
C_{t} & =\mathrm{I}: 8,589,934,592 \\
C_{-t} & =\mathrm{I}: 4.3442+ \\
\left(\mathrm{P}_{4} 6\right) & \\
A & =\mathrm{I} .375+0.5 \mathrm{X} \\
R & =0 \rightarrow \mathrm{I} 2 \\
C t x & =\mathrm{I}: \mathrm{I} 7, \mathrm{I} 79,869, \mathrm{I} 84 \\
C-t x & =\mathrm{r}: 8.6884+ \\
C x & =\mathrm{I}: 2 \\
\left(\mathrm{P}_{4} 7,8, \mathrm{I}\right. & \mathrm{I} 2, \text { and } \mathrm{I} 4) \\
A & =\mathrm{I} .375+0.25 \mathrm{X} \\
R & =0 \rightarrow \mathrm{I} 2 \\
C t x & =\mathrm{I}: 34,359,738,368 \\
C-t x & =\mathrm{I}: 5.7922+ \\
C x & =\mathrm{I}: 4
\end{aligned}
$$

(P4 5 and 16 )

$A=\mathrm{I} .375+0.125 \mathrm{X}$

$R \quad=0 \rightarrow 12$

$C t x=\mathrm{I}: 68,719,476,736$

$C_{-} t x=1: 4.9648+$

$C_{x}=1: 8$ 


\section{For the $F_{1}$ female zygote}

Certain selected formula-values for given ancestors in the $P_{5}$ generation $\left(\mathrm{P}_{5} \mathrm{I}, 2,3,4,5,6,7,8,9,10, \mathrm{I}_{3}, \mathrm{I} 7, \mathrm{I} 8, \mathrm{I9}, 20,2 \mathrm{I}, 25,26\right.$, and 29)

$$
\begin{aligned}
& A=0.6875 \\
& R=0 \rightarrow \mathrm{I} \mathrm{I} \\
& C_{t}=\mathrm{I}: \mathrm{I} 7,592, \mathrm{I} 86,044,4 \mathrm{I} 6 \\
& C_{-t}=\mathrm{I}: 2.033^{2}+
\end{aligned}
$$

$\left(\mathrm{P}_{\mathrm{b}}\right.$ II, I2, I4, and 22)

$$
\begin{aligned}
& A=0.6875+0.25 \mathrm{X} \\
& R=0 \rightarrow \mathrm{I} 2 \\
& C_{t x}=\mathrm{I}: 70,368,744,177,664 \\
& C_{-} t x=\mathrm{I}: 2.7117+ \\
& C_{x}=\mathrm{I}: 4
\end{aligned}
$$

$\left(P_{5} 15,16,23,24,27,28\right.$, and 30$)$

$$
\begin{aligned}
& A=0.6875+0.125 \mathrm{X} \\
& R=0 \rightarrow \mathrm{I} 2 \\
& C t x=\mathrm{I}: \mathrm{I} 40,737,488,355,328 \\
& C_{-t x}=\mathrm{r}: 2.3243+ \\
& C x=\mathrm{I}: 8
\end{aligned}
$$

( $\mathrm{P}_{6} 3^{1}$ and 32$)$

$$
\begin{aligned}
& A=0.6875+0.0625 \mathrm{X} \\
& R=0 \rightarrow \mathrm{I} 2 \\
& C t x=\mathrm{I}: 28 \mathrm{I}, 4744,976,710,656 \\
& C_{t} t x=\mathrm{I}: 2.1693+ \\
& C x=\mathrm{I}: \mathrm{I} 6
\end{aligned}
$$




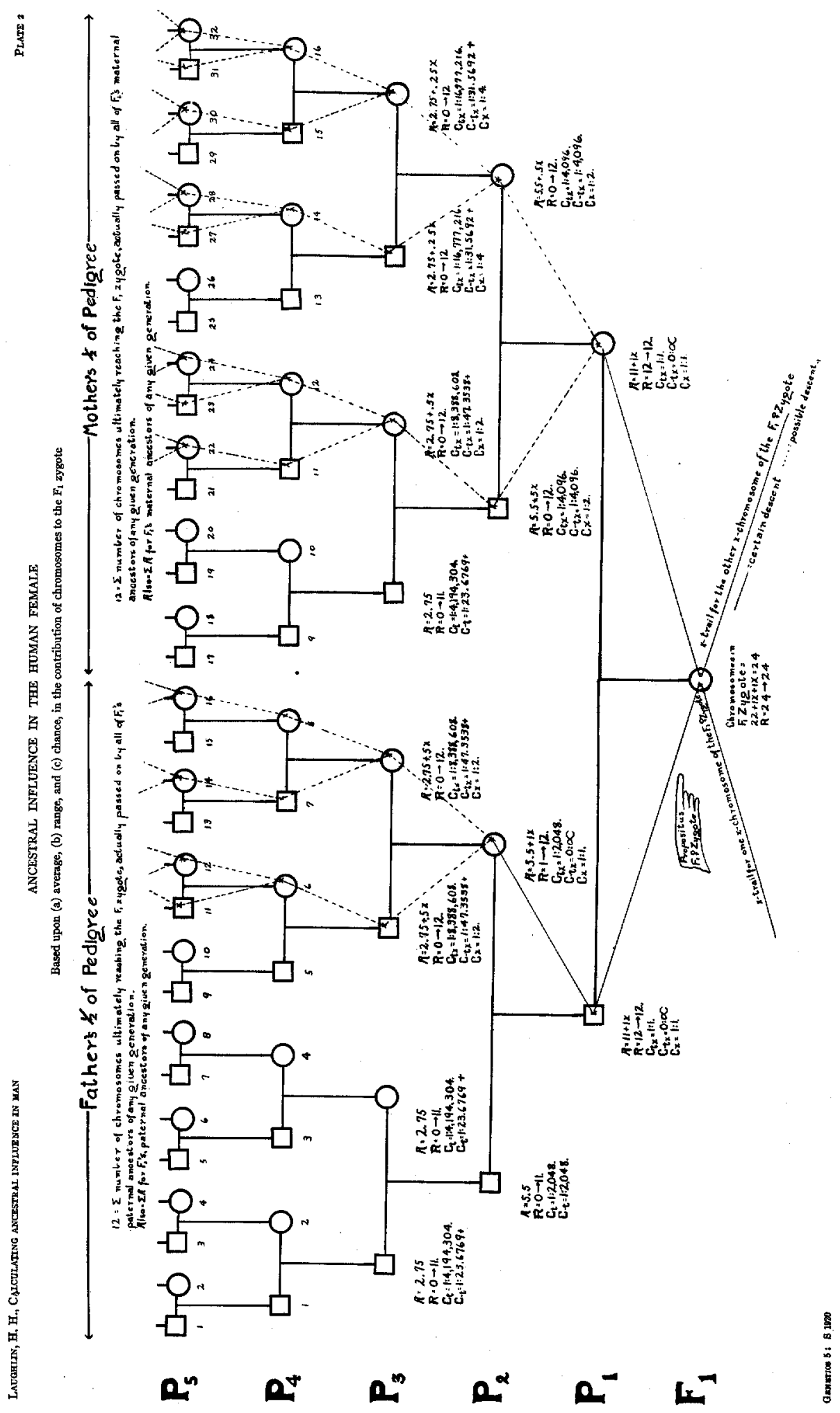

